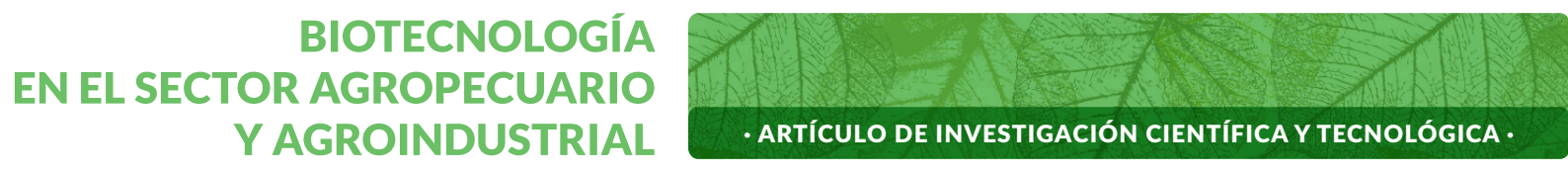

Vol. 18 No 2 · Julio - Diciembre 2020 • ISSN - 1692-3561 · ISSN-e 1909-9959 · DOI: http://dx.doi.org/10.18684

\title{
Actividad antioxidante e influencia del periodo climático sobre el contenido de polifenoles totales en Merremia aegyptia *
}

\section{Antioxidant activity and seasonal influence on total polyphenols content in Merremia aegyptia}

\section{Atividade antioxidante e influência do período climático sobre o conteúdo de polifenóis totais em Merremia aegyptia}

JOSÉ ALBERTO SALGADO-CHÁVEZ" ANDERSON ENRIQUE PALACIO-VALENCIA², NELSON OSVALDO VALERO-VALERO ${ }^{3}$

Historial del Artículo

Recibido para evaluación: 28 de Enero 2020.

Aprobado para publicación: 4 de Mayo 2020.

* Proyecto de investigación de orígen: "Evaluación del potencial antioxidante de dos especies forrajeras (Ipomoea trifida (Kunth) G. Don. y Merremia aegyptia (L.) Urban) presentes en el departamento de La Guajira (Colombia)". Finaciación: Universidad de La Guajira. Culminación: agosto de 2018.

1 Universidad de La Guajira, Facultad de Ciencias Básicas y Aplicadas, Grupo de Investigación Biotecnología. M.Sc. Biotecnología. Riohacha, Colombia. ORCID: https:// orcid.org/0000-0002-8056-0096.

2 Universidad de La Guajira, Programa de Biología, Grupo de Investigación Biotecnología. Riohacha, Colombia. ORCID: https://orcid.org/0000-0003-1505-0599.

3 Universidad de La Guajira, Facultad de Ciencias Básicas y Aplicadas, Grupo de Investigación DESTACAR. Dr. Ciencias Agropecuarias. Riohacha, Colombia. ORCID: https://orcid.org/0000-0001-9186-6245.

Correspondencia: jasalgado@uniguajira.edu.co

Cómo citar este artículo: JOSÉ ALBERTO SALGADO-CHÁVEZ, ANDERSON ENRIQUE PALACIO-VALENCIA, PALACIO-VALENCIA, NELSON OSVALDO VALERO-VALERO. Actividad antioxidante e influencia del periodo climático sobre el contenido de polifenoles totales en Merremia aegyptia. Revista Biotecnología en el Sector Agropecuario y Agroindustrial,18(2),2020.82-93, DOI:http://dx.doi.org/10.18684/BSAA(18)82-93 


\section{RESUMEN}

Merremia aegyptia (Convolvulaceae) no se ve afectada por la aridez que presenta la mayor parte del territorio guajiro (La Guajira, Colombia). Sin embargo, ha sido subestimada y considerada arvense al desconocerse sus propiedades medicinales y otros posibles usos. Así, con la presente investigación se pretendió establecer la influencia de los periodos climáticos y la fenología sobre la producción de polifenoles en dicha especie y estimar el potencial antioxidante de sus extractos. Se tomaron muestras de tejido foliar durante un año y se sometieron a extracción con metanol, la concentración de polifenoles totales fue establecida mediante el método de Folin-Ciacolteau y se correlacionó con la precipitación y temperatura mensual. Además, se estimó el porcentaje de inhibición de los extractos sobre el radical DPPH. Los resultados obtenidos indicaron variación en la concentración mensual de polifenoles totales, siendo enero de 2018 el mes donde se registró la mayor concentración (6,03\%). Adicionalmente, los extractos presentaron mayor actividad antioxidante (85,15\%) que el ácido ascórbico (38,65\%). Se concluyó que la disponibilidad de agua en el suelo afecta la producción de polifenoles en M. aegyptia y que esta especie representa un recurso vegetal susceptible de ser cultivado y explotado bajo las condiciones ambientales de La Guajira.

\section{ABSTRACT}

Merremia aegyptia (Convolvulaceae) is not affected by the predominant aridity in most of guajiro territory (La Guajira, Colombia). However, this species has been underestimated and considered weed since its medicinal properties and other possible uses are unknown. Thus, the present investigation was tried to establish the influence of seasons and the phenology on the production of polyphenols in M. aegyptia and to estimate the antioxidant potential of its extracts. Foliar tissue samples were taken for one year and subjected to methanol extraction, total polyphenol concentration was established by Folin-Ciacolteau method and correlated with monthly precipitation and temperature. In addition the percentage of inhibition of its leaf extracts on the $D P P H$ radical was estimated. Results suggest variation in monthly concentration of total polyphenols, obtaining the highest concentration in January 2018 (6,03\%). Furthermore, the extracts have a higher antioxidant activity $(85,15 \%)$ than ascorbic acid (38,65\%). It was concluded that the availability of water in soil affects the production of polyphenols in M. aegyptia and that this species represents a plant resource capable of being cultivated and exploited under the environmental conditions of La Guajira.

\section{RESUMO}

Merremia aegyptia (Convolvulaceae) não é afetada pela aridez da maior parte do território guajiro (La Guajira, Colômbia). Porém tem sido subestimada e considerada como erva daninha pelo que nao são conhecidas suas propriedades medicinais e outros usos possíveis. Nesse sentido, a presente investigação procurou-se estabelecer a influência dos períodos climáticos e a fenologia na produção de polifenóis nessa espécie e estimar o potencial

\section{PALABRAS CLAVE:}

Bioprospección, Metabolitos secundarios, Convolvulaceae, Precipitación pluvial, Fenología.

\section{KEY WORDS:}

Bioprospection, Secundary metabolites, Convolvulaceae, Rain, Phenology.

\section{PALAVRAS CHAVE:}

Bioprospecção, Metabolitos secundários, Convolvulaceae, Chuvas, Fenologia. 
antioxidante de seus extratos. Amostras do tecido foliar foram colhidas por um ano e submetidas à extração de metanol e, a concentração de polifenóis totais foi estabelecida pelo método de Folin-Ciacolteau e correlacionada com precipitação e temperatura mensais, além da porcentagem de inibição da extratos sobre o radical DPPH. Os resultados obtidos indicaram variação na concentração mensal de polifenóis totais, sendo janeiro de 2018 o mês em que a maior concentração foi registrada (6,03\%); além disso, os extratos apresentaram maior atividade antioxidante (85,15\%) que o ácido ascórbico (38,65\%). Concluiu-se que a disponibilidade de água no solo afeta a produção de polifenóis em $M$. aegyptia e que essa espécie representa um recurso vegetal capaz de ser cultivado e explorado nas condições ambientais do estado La Guajira.

\section{INTRODUCCIÓN}

Muchos de los metabolitos secundarios que producen las plantas presentan actividad biológica, por lo tanto se pueden usar con fines medicinales [1-4]; es así como han hecho parte de la historia de, quizá, todos los grupos étnicos. Actualmente muchas comunidades, indistintamente de si pertenecen a un grupo étnico determinado o no, utilizan las plantas para el tratamiento de una amplia diversidad de dolencias, enfermedades o para controlar los síntomas asociados a las mismas. Pese a que en el departamento de La Guajira (Colombia) la comunidad Wayúu y su legado tienen una alta influencia como resultado de sus prácticas culturales, el conocimiento y utilización de especies vegetales con fines medicinales está disminuyendo, debido, por lo menos en parte, al poco conocimiento de su diversidad biológica. Adicionalmente, los trabajos etnobotánicos son escasos, lo que a su vez limita la proyección de utilización de la biodiversidad vegetal en beneficio de la población del departamento, la región y el mundo.

Históricamente todas las preparaciones medicinales han derivado de especies vegetales [5], ya sea utilizando partes de la planta o extractos crudos de las mismas. Hoy día, muchos medicamentos son desarrollados a partir de plantas que presentan bioactividad y en la mayoría de los casos se requiere el aislamiento de los mismos y su posterior modificación. En la medicina moderna, las plantas son usadas como fuente directa de agentes terapéuticos, modelos para nuevos compuestos sintéticos y como marcadores taxo- nómicos para el descubrimiento de nuevos compuestos [6]. Las batatillas (Convolvulaceae) son plantas forrajeras que podrían aprovecharse en la obtención de sustancias bioactivas [7-10]; no obstante, a estas plantas se les considera hierbas invasoras de terrenos no ocupados e incluso han sido reportadas como arvenses y nocivas para el ganado [11-13], desestimando su valor ecológico, nutricional o medicinal.

La obtención de nuevas sustancias para el tratamiento de diversas enfermedades es limitada y la información básica para la elaboración de fármacos menos costosos y con menos efectos secundarios es aún escasa y requiere de la evaluación de materiales que puedan producir de manera sostenida grandes cantidades de compuestos activos de manera controlada. A pesar de que existen metodologías para la extracción y cuantificación de polifenoles a partir de distintos órganos vegetales [14-20] la concentración de este tipo de sustancias depende mucho de la variedad, la fenología, la localización geográfica y la época de colecta, además el contenido de metabolitos secundarios es variable entre una planta y otra, aunque pertenezcan a la misma variedad $[6,21]$. En este sentido, varias especies del género Merremia han sido objeto de investigación en relación con el contenido de polifenoles y su potencial antioxidante, tal es el caso de M. borneensis Merr. [22], M. hederacea (Burm. f.) Hallier f., M. gangitica (L.) Cufo. [23], M. emarginata (Burm. F.) Hall. F. [24], M. mammosa (Lour.) Hallier f. [25] y M. umbellata (L.) H. Hallier [26]. Así mismo, el uso tradicional de M. aegyptia como antidiabético, cicatrizante, antimicrobiano y antitumoral ha sido reportado [6], lo cual puede estar relacionado con su contenido de polifenoles.

La concentración de fenoles en tejidos vegetales se ha utilizado como indicador del grado de resistencia al estrés [27] y específicamente a los polifenoles se les ha atribuido propiedades medicinales entre las que se destacan su actividad antialergénica, antioxidante, anticancerígena, antihiperglicémica, antimicrobiana y antiinflamatoria [28-32], esto indica la necesidad de conocer los beneficios de la biodiversidad vegetal presente en el territorio guajiro con miras a un mejor aprovechamiento, y aunque una revisión bibliográfica podría mejorar el conocimiento sobre la utilización de las especies vegetales presentes en la región, la proyección de futuras investigaciones y la obtención de bioproductos de interés comercial sólo se logrará con la realización de trabajos que permitan 
identificar los metabolitos que estas especies producen y qué factores ambientales influyen sobre la producción de este tipo de sustancias. La especie objeto de estudio de la presente investigación tiene la ventaja adicional de estar totalmente adaptada a ecosistemas áridos [33, 34], lo que implica disponibilidad de material para trabajar, en caso de comprobarse la alta producción de sustancias bioactivas bajo las condiciones ambientales de La Guajira.

Con la presente investigación se buscó estimar el potencial antioxidante de los extractos foliares de M. aegyptia y determinar las variaciones en la concentración de polifenoles atendiendo a los periodos climáticos regentes en región Caribe colombiana y a la fenología de la especie. La información generada soporta las bases para proponer el cultivo de $M$. aegyptia, así como la posibilidad de estimar la mejor época de cosecha considerando el contenido de polifenoles en las hojas y de esta manera aprovechar la disponibilidad de suelos que por su aridez no permiten el establecimiento de cultivos convencionales.

\section{MÉTODO}

\section{Muestreo y definición de los periodos climáticos}

Se tomaron muestras de plantas de M. aegyptia, visiblemente sanas y sin signos de herbivoría que crecían en estado silvestre en un área urbana del municipio de Riohacha (La Guajira), en las coordenadas $11^{\circ} 32^{\prime} 15,7^{\prime \prime}$ de latitud Norte y $72^{\circ} 53^{\prime} 36,7^{\prime \prime}$ de longitud Oeste, con una temperatura promedio de 29,24 $\pm 1,31^{\circ} \mathrm{C}$ y humedad relativa de $72,75 \pm 4,11 \%$. Las muestras consistieron de hojas procedentes de la parte media de los tallos, las cuales fueron tomadas mensualmente durante un año, en el periodo comprendido entre septiembre de 2017 y agosto de 2018. Con el fin de establecer la concentración de polifenoles en otros órganos, en el mes de marzo de 2018 se colectaron flores completas (incluían todos los órganos florales) y frutos maduros, en perfecto estado, procedentes de las plantas objeto de estudio. Posterior a la colecta, las muestras se trasladaron inmediatamente al Laboratorio de Calidad Ambiental de la Universidad de La Guajira (Riohacha, Colombia), donde fueron procesadas para la obtención de extractos vegetales.

Durante el periodo de estudio se registraron los cambios fenológicos que presentaron las plantas objeto de estudio y se establecieron tres estadíos fenológicos: Crecimiento vegetativo (ausencia de estructuras reproductivas), Floración (desde la aparición de las primeras yemas florales hasta la aparición de los primeros frutos) y Fructificación (a partir de la aparición de los primeros frutos). Los periodos climáticos fueron definidos utilizando los reportes históricos y los datos climatológicos (precipitación y temperatura) correspondientes al periodo evaluado en la presente investigación, publicados por el Instituto de Hidrología, Meteorología y Estudios Ambientales IDEAM [35].

Adicionalmente, se tomaron por duplicado muestras del horizonte A del suelo adyacente a las plantas y se hicieron determinaciones de los siguientes parámetros: densidad aparente (método núcleo), textura (Boyucos), humedad gravimétrica (gravimetría), estructura (manual), retención de humedad a 0,33 atm (plato de Richards), $\mathrm{pH}$ (potenciométrico), conductividad eléctrica (conductímetro); siguiendo los protocolos de la sección de física de suelos del laboratorio de Ciencias Ambientales de la Universidad de La Guajira. Al finalizar el muestreo las raíces de las plantas evaluadas fueron extraídas y medidas en miras a asociar las estrategias adaptativas de la especie para superar el déficit hídrico con el contenido de polifenoles.

\section{Obtención de los extractos foliares}

Hojas procedentes de tres plantas de $M$. aegyptia fueron secadas en horno durante $48 \mathrm{~h}$ a $40^{\circ} \mathrm{C}$, posteriormente se maceraron independientemente en nitrógeno líquido a temperatura de laboratorio $\left(24 \pm 1^{\circ} \mathrm{C}\right)$, por aproximadamente $5 \mathrm{~min}$, hasta obtener un polvo fino. Se pesó $1 \mathrm{~g}$ de cada pulverizado y se les adicionó $10 \mathrm{~mL}$ de metanol al 96\%, se dejó actuar por 2 horas y se filtró utilizando papel filtro Whattman $\mathrm{N}^{\circ} 4$, siguiendo la metodología descrita en trabajos previos [36]. Los extractos así obtenidos fueron utilizados en los ensayos posteriores.

\section{Cuantificación de polifenoles totales}

La cuantificación de polifenoles se hizo de acuerdo con lo establecido por la IAEA [37], utilizando un espectrofotómetro UV-Vis Hach DR 5000. Los límites de detección y cuantificación del equipo fueron estimados como se describe en la guía tripartita armonizada de la International conference on harmonisation of technical requirements for registration of pharmaceuticals for human use [38], mediante el 
método basado en la desviación estándar del blanco y la pendiente de una curva de calibrado de ácido gálico construida para tal fin.

Se tomaron tres alícuotas de $21 \mu \mathrm{L}$ de cada extracto y se depositaron en tubos de ensayo, de manera independiente se les adicionaron agua destilada hasta un volumen de $1,25 \mathrm{~mL}$, se les adicionó $625 \mu \mathrm{L}$ del reactivo de Folin-Ciocalteu, se dejaron reposar por 10 minutos y luego se les adicionaron $3,125 \mathrm{~mL}$ de solución de $\mathrm{Na}_{2} \mathrm{CO}_{3}$ al $20 \%$. La mezcla en los tubos de ensayo fue agitada en vortex hasta homogenizar, después de dos horas se midió la absorbancia a $760 \mathrm{~nm}$. Los valores de absorbancia se utilizaron para establecer la concentración de polifenoles totales utilizando la ecuación de la curva de calibración $\left(y=0,0458 x+0,0092 ; R^{2}=0,9998\right)$ y el contenido de estas sustancias se expresó en porcentaje sobre una base de masa seca ( $1 \mathrm{~g}$ de tejido pulverizado), como equivalente de ácido gálico (\%EAG). Los órganos reproductivos fueron procesaron de manera similar al tejido foliar.

\section{Actividad antioxidante}

La actividad antioxidante de los extractos se determinó mediante el método del DPPH [1], como se describe a continuación. Se tomaron cinco alícuotas de $100 \mu \mathrm{L}$ de cada extracto y se mezclaron, independientemente, con 3,9 mL de una solución de 1,1-Difenil-2-picrílhidrazilo (DPPH) a una concentración de $60 \mu \mathrm{mol}$, recién preparada en metanol al 96\%; la mezcla se incubó a temperatura de laboratorio (24 \pm $1^{\circ} \mathrm{C}$ ) en la oscuridad y se midió la absorbancia a 515 $\mathrm{nm}$ a los 0 y 30 minutos de reacción. Se utilizó metanol al 96\% como control negativo y soluciones de ácido ascórbico a la misma concentración de polifenoles totales estimada en los extractos como control positivo. Los porcentajes de inhibición del radical DPPH se calcularon de acuerdo con la ecuación 1[39]:

$$
\% \text { Inhibición }=\frac{A b s_{D P P H}-A b s_{\text {muestra }}}{A b s_{D P P H}}
$$

Dónde: $\mathrm{Abs}_{\mathrm{DPPH}}$ es la absorbancia del control negativo a $\mathrm{t}=0$ minutos y $\mathrm{Abs}_{\text {muestra }}$ es la absorbancia de las muestras a $\mathrm{t}=30$ minutos.

\section{Procesamiento de datos y análisis de la información}

Todos los ensayos se ajustaron a un diseño completamente aleatorizado. Los datos correspondientes a la concentración mensual de polifenoles y actividad antioxidante fueron tabulados y graficados en Microsoft Excel ${ }^{\circledR}$, analizados con ANAVA y las medias fueron separadas mediante el test de Duncan. La relación entre la fenología y el contenido de polifenoles se estableció comparando las variaciones en la concentración mensual de estas sustancias contra el estadío fenológico de las plantas en el mismo periodo; por su parte, la concentración mensual de polifenoles fue correlacionada con la temperatura media mensual y las precipitaciones mediante el coeficiente de correlación de Pearson. Todos los análisis estadísticos fueron realizados utilizando el paquete de análisis estadístico $\mathrm{SAS}^{\circledR} \mathrm{v}$. University.

\section{RESULTADOS}

\section{Fenología de las plantas, características del suelo y periodos climáticos}

Durante el periodo de estudio las plantas evaluadas presentaron la siguiente fenología: Crecimiento vegetativo, de septiembre a mediados de diciembre de 2017; Floración, mediados de diciembre de 2017 hasta mediados de enero de 2018. El estadío de Fructificación se dio desde mediados de enero de 2018 y se prolongó con la presencia simultánea de flores durante el resto del tiempo contemplado en la investigación (agosto de 2018). Los cambios fenológicos coinciden con los descritos para dicha especie [34], resaltando que esta planta, propia de ambientes secos, florece y fructifica mayoritariamente durante los meses de octubre a enero y en menor grado en otros meses del año por influencia de las precipitaciones. Sin embargo, no fue posible establecer la relación entre el contenido de polifenoles y la fenología de las plantas, ya que la concentración de polifenoles varió indistintamente del estadio reproductivo de las plantas, por lo que se consideró que la producción de este tipo de sustancias se ve afectada mayoritariamente por las variables ambientales y no por el estadio fenológico.

El análisis del suelo adyacente a las plantas reveló una densidad de $1,45 \mathrm{~g} / \mathrm{cm}^{3}$, lo que indica un alto grado de compactación que puede conllevar a dificultades para la circulación del agua y el desarrollo de raíces; la 
estructura blocosa angular con desarrollo moderado a fuerte y bloque de tamaño medio de $19 \mathrm{~mm}$ es típica en suelos donde la actividad biológica y la materia orgánica no tienen gran influencia como factor formador; la textura franco limosa con contenidos de arcilla de $26,5 \%$, limo del $37 \%$, y arena del 36,55 es coherente con el grado de estructura presente y la baja conductividad y retención hídrica, reflejada en una humedad gravimétrica del $4,96 \%$ que indica un déficit hídrico alto y la baja capacidad de retención de humedad a 0,33 atm (11,3\%); también, se evidenció alcalinidad $(\mathrm{pH} 8,48)$ y baja conductividad eléctrica (1156 $\mu \mathrm{S}$ ). Las características predominantes son congruentes con lo reportado para los suelos de la zona [40] y en su conjunto estos parámetros edáficos pueden representar condiciones estresantes para el desarrollo vegetal, más aún bajo las condiciones climáticas predominantes. Por su parte, las raíces de las plantas utilizadas durante la investigación tuvieron una longitud promedio de $163,9 \mathrm{~cm}( \pm 40,46 \mathrm{~cm})$ y se extendieron hasta una profundidad de $75,5 \mathrm{~cm}( \pm 14,08 \mathrm{~cm})$.

En cuanto a los periodos climáticos, hubo correspondencia entre el régimen de lluvias evidenciado durante el periodo evaluado y los registros históricos [35], presentándose dos periodos lluviosos: el primero se extendió desde el mes de septiembre hasta noviembre de 2017, siendo octubre y noviembre los meses con mayores precipitaciones (180 y $164 \mathrm{~mm}$, respectivamente) con algunas Iluvias esporádicas en el mes de diciembre. Durante este primer periodo se obtuvo un acumulado de $399 \mathrm{~mm}$, concentrando la mayor precipitación del año. El segundo periodo de lluvias se dio entre los meses de abril a junio de 2018 , siendo las lluvias menos intensas y abundantes ( $43,7 \mathrm{~mm}$ en total). Los periodos secos se dieron durante los meses de enero, febrero, marzo, julio y agosto de 2018 , destacándose por precipitaciones muy bajas (entre $0,5 \mathrm{~mm}$ en el mes de marzo de 2018 y $16,3 \mathrm{~mm}$ en el mes de agosto del mismo año) y temperaturas más altas durante los meses de julio y agosto $\left(31,22 \pm 0,01^{\circ} \mathrm{C}\right)$. No obstante lo anterior, se resalta que el terreno adyacente al sitio donde crecieron las plantas objeto de estudio permaneció inundado por varios meses (octubre de 2017 a enero de 2018), evento que fue atribuido a las características físicas del suelo, las cuales dificultaron la conductividad hidráulica que permitiera la percolación del agua del período lluvioso (septiembre noviembre de 2017), lo que pudo haber influido en la concentración de polifenoles en algunos meses de la temporada seca.

\section{Concentración de polifenoles totales en los extractos foliares}

El límite de detección del espectrofotómetro utilizado fue estimado en 0,41 ppm y el límite de cuantificación en 1,24ppm.

Los resultados obtenidos evidenciaron que la concentración de polifenoles en M. aegyptia varían de un mes a otro durante todo el año y el análisis estadístico de los datos corroboró la existencia de diferencias significativas en el comportamiento de esta variable durante el periodo evaluado $(p<0,0001)$. Se registró un incremento paulatino en la producción de polifenoles totales desde los meses de septiembre de 2017 (0,32\% EAG) a enero de 2018 (6,03\% EAG); posteriormente se evidenció un descenso en la concentración de estas sustancias durante los meses de febrero y marzo de 2018 (1,24\% EAG en ambos meses), seguido de un leve aumento durante los meses de

Figura 1. Variación mensual del contenido promedio de polifenoles totales (\%) en M. aegyptia (barras \pm la desviación estándar). La línea continua muestra la precipitación mensual (mm).

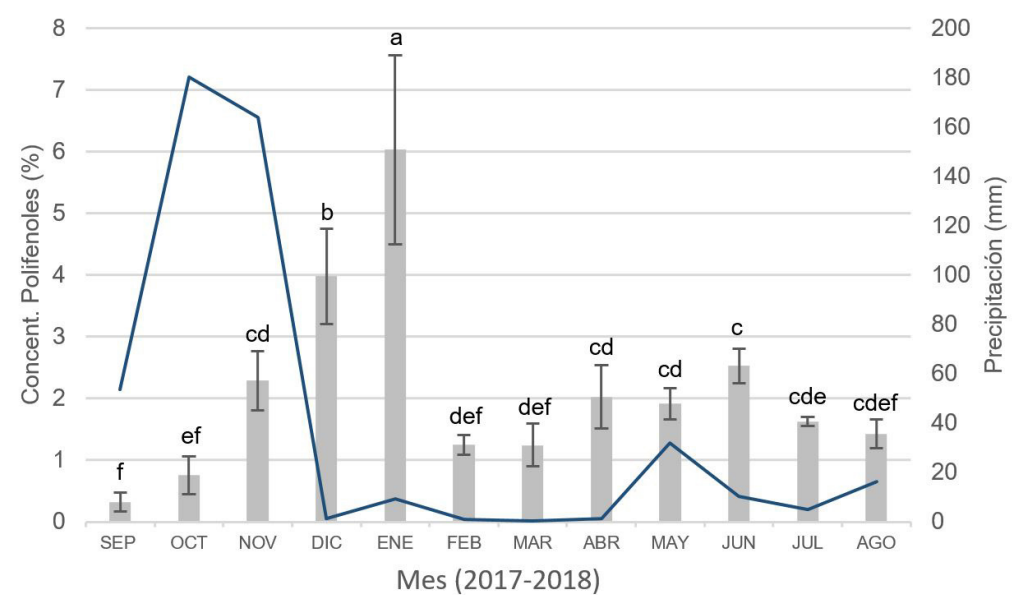

Letras diferentes indican diferencias estadísticamente significativas entre el contenido de polifenoles $(a=0,05)$. 
abril a junio de 2018 (2,02 a 2,52\% EAG), disminuyendo nuevamente durante los meses de julio y agosto (1,62 y $1,42 \%$ EAG, respectivamente) (figura 1$)$.

Debido a que en los meses de diciembre de 2017 y enero de 2018 se evidenció la mayor concentración de las sustancias de interés (siendo estos parte de uno de los periodos secos del año y se esperaba que la concentración de polifenoles en las plantas evaluadas disminuyera), no se encontró correlación significativa entre el contenido de polifenoles y el régimen de lluvias ( $r=-0,2676 ; p=0,4004)$. Aun así, varios trabajos de investigación señalan la relación existente entre estas dos variables y se considera coherente con los resultados de la presente investigación teniendo en cuenta que había agua acumulada en el suelo durante los primeros meses del periodo seco. De igual manera, no se evidenció correlación entre la temperatura mensual promedio y la concentración mensual de polifenoles $(r=-0,1836 ; p=0,5678)$.

El incremento en el contenido de polifenoles desde el mes de noviembre de 2017 hasta enero de 2018 se atribuyen, por un lado, a las lluvias relativamente frecuentes durante los meses de septiembre a noviembre de 2017, así como a la inundación del área colindante con el sitio donde crecían las plantas, la cual se mantuvo desde el mes de octubre de 2017 hasta entrado el año 2018, posiblemente como consecuencia de las características físicas del suelo. Como se indicó en párrafos anteriores, los meses de diciembre a febrero corresponden históricamente al periodo seco; sin embargo, el alto contenido de polifenoles en estos meses se considera que estuvo influenciado por la acumulación de agua en la superficie del suelo y su lenta infiltración como consecuencia de la compactación del mismo. Sumado a su poca estructura (debida a la falta de materia orgánica), los bloques que conforman este suelo presentaron resistencia a romperse y tuvieron un grado fuerte de desarrollo, características que dificultan la capacidad de las raíces de las plantas para explorar horizontalmente; no obstante, las raíces presentaron una longitud considerable y una penetración suficiente como para acceder al agua contenida en estratos bajos del suelo, lo cual proporcionó mayor cantidad de agua a estas plantas, incrementado la producción de polifenoles.

Se ha descrito el efecto de la cantidad de agua disponible para las plantas sobre la producción de polifenoles en Camellia sinensis L. [41], encontrando que el aumento en el contenido de agua en el suelo tiene una relación directamente proporcional con la concentración de polifenoles, variando desde $12 \%$ en suelos con el menor potencial hídrico ( $14 \%$ de contenido de agua) hasta $25 \%$ en los suelos con mayor potencial hídrico (38\% de contenido de agua). Estos hallazgos fueron soportados en el hecho de que al ser el agua uno de los insumos de la fotosíntesis, afecta la producción de sustancias orgánicas en la planta, entre estas los metabolitos secundarios. Los resultados en mención coinciden con los obtenidos en Olea europaea L. Cv. Cobrançosa (un genotipo bien adaptado a ambientes con bajo suministro anual de agua), donde se evidenció que la deficiencia de agua disminuye el contenido de polifenoles, siendo muy evidente en condiciones de secano (disminución en el contenido

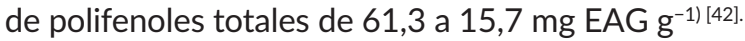

Los menores contenidos de polifenoles en M. aegyptia durante los meses de septiembre-octubre de 2017 y febrero-marzo de 2018 sugieren una respuesta a la falta de agua en los meses de sequía que los precedieron (julio - agosto y diciembre-enero, respectivamente), disminuyendo la producción de compuestos fenólicos. Así mismo, el incremento en la concentración de estas sustancias en los meses de abril a junio de 2018 coincide con las lluvias registradas en este periodo. La influencia de la variación estacional sobre el contenido de polifenoles en hojas de I. batatas ha sido establecida [43], hallando que la concentración de éstos disminuye durante el verano e incrementaba nuevamente después de dicha estación. Así mismo, se halló una concentración promedio de polifenoles de $6,9 \%$, lo cual contrasta con lo encontrado en la presente investigación al obtenerse una producción promedio de $2,11 \%$ EAG en M. aegyptia. No obstante, en el trabajo anteriormente citado no se correlacionó la disponibilidad de agua para las plantas con la producción de polifenoles, en su lugar se encontró que esta variable se correlacionaba directamente (y de forma negativa) con la temperatura ambiental, lo cual difiere con los resultados de la presente investigación, donde no se evidenció correlación entre estas variables.

M. aegyptia es una especie adaptada a condiciones de aridez, por tanto se esperaba que mantuviera altos contenidos de polifenoles como estrategia contra el estrés ambiental, como ha sido evidenciado en Achillea pachycephala y Sesamum indicum [44, 45], donde se encontró que la concentración de polifenoles incrementa a medida que las plantas se exponen 
a mayores niveles de estrés hídrico. Por otro lado, en Brassica rapa [46] se encontró que el estrés hídrico disminuye la concentración de polifenoles de forma leve, lo cual deja en evidencia que el genotipo influye sobre esta respuesta fisiológica. Los trabajos realizados sobre las especies anteriormente citadas reportan que los flavonoides decrecen bajo condiciones de estrés, mientras que los ácidos caféico y clorogénico incrementan, coincidiendo con los resultados obtenidos en Cynara cardunculus [47].

Se ha establecido que el estrés hídrico incrementa el contenido de polifenoles totales en Amaranthus tricolor, (una especie adaptada a dicho estrés) [48] y existen reportes de la producción variable de polifenoles en especies que crecen en un ecosistema árido de Brasil; oscilando entre 2,5\% (Macroptilium lathyroides (L) Urban) y 22,0\% (Stylosanthes humilis Kunth) [49], lo que supone la influencia del genotipo y las adaptaciones al estrés sobre el comportamiento de esta variable. También se reporta una producción de polifenoles de 6,1\% en M. aegyptia, valor similar al encontrado en el presente trabajo para esta especie en el mes de enero de 2018 bajo las condiciones ambientales de La Guajira; sin embargo, debe tenerse en cuenta que los datos tomados en los trabajos aquí citados son puntuales y no consideran una línea de tiempo, lo que no permite evidenciar variaciones en el contenido de estas sustancias. Así, la diferencia en el contenido de polifenoles durante el resto del año puede deberse a las variaciones en las condiciones ambientales a las que estuvieron sometidas las plantas y corresponden con los periodos climáticos.

El contenido de polifenoles totales en seis genotipos de $I$. batatas fue analizado, encontrando que la concentración de éstos fluctuó entre 12,18 y 16,17\% [14]. Posteriormente, se indicó que en esta misma especie el contenido de polifenoles varía entre 6,3 y $13,5 \%$ (dependiendo del genotipo) [36], valores que distan de los hallados en el presente trabajo; no obstante, debe resaltarse que M. aegyptia crece abundantemente en suelos marginales del territorio guajiro, por lo que aun así representa un buen insumo para la producción de antioxidantes que merece ser estudiada a fondo con fines de aprovechamiento. Además, las propiedades medicinales de M. aegyptia y otras especies relacionadas han sido reportadas, destacándose su utilización como antimicrobiano, diurético, tratamiento de quemaduras en la piel y trastornos del hígado [2, 50, 51], bioactividad que también ha sido asociada con la presencia de polifenoles.

La concentración promedio de polifenoles en flores fue de $7,4 \%( \pm 1,05)$, mientras que la concentración de estas sustancias en los frutos estuvo por debajo del límite de detección del equipo. En relación con la concentración de polifenoles en tejido foliar en el mes de marzo de 2017 (1,24 $\pm 0,34 \%)$, las flores presentaron contenido de polifenoles 5,94 veces superior. La diferencia en el contenido de polifenoles en hojas y flores sugieren que los polifenoles producidos en las hojas son traslocados a las flores donde desempeñarán funciones relacionadas con la atracción de polinizadores o evitar la florivoría [52, 53]. A su vez, la mayor concentración de polifenoles en flores explicaría, en parte, los valores marcadamente más bajos de estas sustancias en las hojas durante los estadios de floración y fructificación.

\section{Actividad antioxidante}

Los resultados de la presente investigación mostraron que la capacidad antioxidante de los extractos foliares de M. aegyptia (85,15\%) es superior a la del ácido ascórbico (38,65\%), lo cual es atribuido a la concentración de polifenoles en los mismos. El análisis estadístico de los datos permitió establecer diferencias estadísticamente significativas en el porcentaje de inhibición del radical DPPH para las sustancias evaluadas ( $<<0,0001$ ) (Cuadro 1 ).

Cuadro 1. Actividad antioxidante de extractos de M. aegyptia sobre una solución de DPPH (60 $\mu$ molar). Los valores en cada celda representan la media de cada tratamiento \pm la desviación estándar.

\begin{tabular}{|c|c|c|c|}
\hline MUESTRA & ABS 0' & ABS 30' & \% INHIBICIÓN 30' \\
\hline Extracto M. aegyptia (38,93 ppm) & $0,110 \pm 0,006$ & $0,103 \pm 0,006$ & $85,15 \pm 0,79$ a \\
\hline Ác. Ascórbico (38,93 ppm) & $0,428 \pm 0,002$ & $0,427 \pm 0,002$ & $38,65 \pm 0,28 \mathrm{~b}$ \\
\hline Met-OH 96\% & $0,696 \pm 0,000$ & $0,695 \pm 0,001$ & $0,215 \pm 0,10$ \\
\hline
\end{tabular}

Letras diferentes indican diferencias estadísticamente significativas entre los porcentajes de inhibición de las sustancias evaluadas sobre el radical DPPH $(a=0,05)$. 
Los resultados tabulados en el Cuadro 1 son similares a los obtenidos en trabajos previos [23], donde se reporta la inhibición del radical DPPH en un 60,31\% utilizando extractos foliares de M. gangitica, haciendo énfasis en que la actividad antioxidante de los extractos fue superior a aquella obtenida con soluciones de ácido ascórbico a $100 \mathrm{mg} \mathrm{mL}^{-1}$, lo cual fue relacionado con la concentración de polifenoles en los extractos. Así mismo, se ha obtenido correlación positiva alta $\left(R^{2}=0,907\right)$ entre la concentración de polifenoles en extractos foliares de I. batatas y la actividad antioxidante de los mismos [36], lo que coincide con otros reportes $[39,54,55,56]$, donde se señala la relación entre un mayor contenido de compuestos fenólicos y la alta actividad antioxidante de extractos vegetales. Por otra parte, la relación directa entre el contenido de derivados de ácidos fenólicos y flavonoides con la actividad antioxidante de extractos crudos de M. borneensis ha sido argumentada (hasta un $80 \%$ de inhibición del radical DPPH, utilizando $100 \mu$ g de extracto) [22].

No obstante lo anterior, se hace necesario conocer el tipo y concentración individual de los compuestos fenólicos presentes en los extractos más que la concentración total de éstos, ya que este grupo de sustancias es bastante amplio y varios autores [1, 43, 57] han señalado la presencia de derivados del ácido cafeoilquinico, ácido gálico, ácido cinámico, ácido ferúlico, eriocitrina, hesperidina, ácido rosmarínico y metil-rosmarenato en extractos vegetales, todos ellos con diferente potencial antioxidante. De igual manera, se ha encontrado diferencias en la actividad antioxidante al evaluar fracciones de los extractos de $M$. borneensis [22], lo que refuerza la idea anterior. Por otro lado, se encontró que en I. batatas la composición y concentración de polifenoles individuales varía entre genotipos de la misma [14], lo cual podría presentarse a nivel individual y que también es susceptible de estar influenciado por variables ambientales.

Aunque algunos autores consideran que los polifenoles son las sustancias con las mayores propiedades antioxidantes en organismos vegetales [36, 58], otros reconocen que estos compuestos no son los únicos fitoconstituyentes en un extracto vegetal que pueden inhibir radicales libres [39]. Sobre este hecho, se ha señalado que aparte de los polifenoles, la vitamina $\mathrm{E}$, el ß-caroteno y la luteína pueden contribuir a la inhibición de radicales libres en extractos foliares de l. batatas [36], por lo que no podría descartarse que otras sustancias presentes en los extractos de M. aegyptia hayan contribuido a los resultados obtenidos. Sin embargo, también debe considerarse que la actividad antioxidante de los extractos vegetales (y por extensión el de otras sustancias) puede estar influenciada por los diferentes mecanismos de inhibición de radicales libres que presentan las sustancias que los constituyen, asociados con algunas variables como peso molecular, número de anillos aromáticos y naturaleza de la sustitución de grupos hidroxilo [28]. Adicionalmente, se ha reportado a los ácidos cafeoilquínicos como los compuestos con mayor concentración en las hojas de I. batatas y que esta varía entre periodos climáticos [43], lo que influye sobre la capacidad que tienen los extractos de inhibir radicales libres, dejando en evidencia que la época en que se cosechen las hojas para obtener los extractos definirá, por lo menos en parte, el grado de actividad antioxidante de los mismos.

\section{CONCLUSIONES}

M. aegyptia constituye una fuente potencial de polifenoles y la disponibilidad de agua para las plantas de esta especie (y no la fenología, ni la temperatura) determina la concentración de este tipo de sustancias, por lo que la época del año (considerando específicamente las precipitaciones) o la saturación de agua que tenga el suelo son factores a tener en cuenta al momento de planear la cosecha de las plantas si se pretende su aprovechamiento industrial. Así mismo, se requiere la cuantificación individual de polifenoles para garantizar que la extracción se realice en el momento donde aquellos que presenten mayor actividad antioxidante estén presentes en concentraciones relativamente altas.

\section{REFERENCIAS}

[1] HAMDY, M., ATEF, M., SELIM, K. and IBRAHIM, K. Evaluation of antioxidant activity, total phenols and phenolic compounds in thyme (Thymus vulgaris L.), sage (Salvia officinalis L.), and marjoram (Origanum majorana L.) extracts. Industrial Crops and Products, 43, 2013, p. 827- 831. doi: https:// doi.org/10.1016/j.indcrop.2012.08.029.

[2] JOSHI, R., AGARWAL, S. and PATNI, V. Evaluation of anti-microbial activity of in vitro and in vivo plant parts of Merremia Dissecta and Merremia Aegyptia. International Journal of Pharmaceutical Sciences and Research, 6(6), 2015, p. 2477-2483. doi: 10.13040/IJPSR.0975-8232.6(6).2477-83. 
[3] TUNGMUNNITHUM, D., THONGBOONYOU, A., PHOLBOON, A. and YANGSABAI, A. Flavonoids and other phenolic compounds from medicinal plants for pharmaceutical and medical aspects: An Overview. Medicines (Basel, Switzerland), 5(3), 93, 2018. doi: https://doi.org/10.3390/ medicines5030093.

[4] HUSSEIN, R. and EL-ANSSARY, A. En: Herbal Medicine. Plants secondary metabolites: the key drivers of the pharmacological actions of medicinal plants. London (United Kingdom): Intechopen Limited, 2018, p. 11-30. doi: 10.5772/intechopen.76139.

[5] CATALANO, E. En: Studies in Natural Products Chemistry. Chapter 3 - Nanotechnology-Based Drug Delivery of Natural Compounds and Phytochemicals for the Treatment of Cancer and Other Diseases. Cambridge (United States): Elsevier, 2019, p. 91-123. doi: https://doi.org/10.1016/B978-0444-64185-4.00003-4.

[6] OMOTAYO, F. and BOROKINI, T. Comparative phytochemical and ethnomedicinal survey of selected medicinal plants in Nigeria. Scientific Research and Essays, 7(9), 2012, p. 989-999. doi: 10.5897/SRE09.525.

[7] AMBIKA, A. and NAIR, S. Wound healing activity of Plants from the Convolvulaceae family. Advances in Wound Care, 8(1), 2019, p. 28-37. doi:10.1089/ wound.2017.0781.

[8] JOSHI, R., MEENA, R. and PATNI, V. Comparative phytochemical analysis of bioactive constituents present in in vitro and in vivo plant parts of $\mathrm{Me}$ rremia aegyptia and Merremia dissecta. Journal of Pharmacognosy and Phytochemistry, 7(1), 2018, p. 679-684. doi: http://dx.doi.org/10.22271/phyto.

[9] CHEN, G., LU, Y., YANG, M., LI, J. and FAN, B. Medicinal uses, pharmacology, and phytochemistry of Convolvulaceae plants with central nervous system efficacies: A systematic review. Phytotherapy Research, 32(5), 2018, p. 823864. doi:10.1002/ptr.6031.

[10] SALEHI, B. et al. Convolvulus plant-A comprehensive review from phytochemical composition to pharmacy. Phytotherapy Research, 34(2), 2019, p. 237-431. doi:10.1002/ptr.6540.

[11] PACHECO, D. et al. Reconocimiento de malezas presentes en el huerto de guayabo (Psidium guajava L.) tipo Criolla Roja, del Centro Frutícola del Zulia, Municipio Mara, Venezuela. Revista UDO Agrícola, 9(1), 2009, p. 141-147.

[12] BIDÓIA, V. Estratégias de manejo químico para controle de Mucuna aterrima, Ricinus communis,
Merremia aegyptia, Ipomoea hederifolia e Ipomoea quamoclit e sua seletividade no plantio da cana-de-açúcar [Mestre em Agronomia (Produção Vegetal)]. Jaboticabal (Brasil): Universidade Estadual Paulista - UNESP, Faculdade de Ciências Agrárias e Veterinárias, 2019, 72 p.

[13] BRITO, L. et al. Spontaneous and experimental poisoning by Merremia macrocalyx (Convolvulaceae) in cattle. Pesquisa Veterinária Brasileira, 39(7), 2019, p. 447-453. doi: https://doi.org/10.1590/16785150-pvb-6335.

[14] ISLAM, I., SHAIKH, A. and SHAHIDUL, I. Antioxidative and antimutagenic potentials of phytochemicals from Ipomoea batatas (L) Lam. Internacional Journal of Cancer Research, 5(3), 2009, p. 83-94. doi: 10.3923/ijcr.2009.83.94.

[15] KOCZKA, N., STEFANOVITS-BÁNYAI, É. and OMBÓDI, A. Total polyphenol content and antioxidant capacity of rosehips of some Rosa species. Medicines, 5(3), 2018, 84. doi:10.3390/ medicines 5030084.

[16] ZHANG, H. et al. Effect of solvents on polyphenol recovery and antioxidant activity of isolates of Asparagus Officinalis roots from Chinese and New Zealand cultivars. International Journal of Food Science \& Technology, 53(10), 2018, p. 2369-2377. doi:10.1111/ijfs.13829.

[17] ABDELOUHAB, K. et al. Comparative study of the polyphenol content related-antioxidant and anti-inflammatory activities of methanolic extracts from different parts of Hertia cheirifolia. International Journal of Pharmaceutical Research, 11(4), 2019, p. 209-215. doi: https://doi. org/10.31838/ijpr/2019.11.04.034.

[18] BAZZICALUPO, M. et al. Polyphenol characterization and skin-preserving properties of hydroalcoholic flower extract from Himantoglossum robertianum (Orchidaceae). Plants, 8(11), 2019, 502. doi: $10.3390 /$ plants 8110502 .

[19] GULCIN, I. et al. Anticholinergic, antidiabetic and antioxidant activities of cinnamon (Cinnamomum verum) bark extracts: polyphenol contents analysis by LC-MS/MS. International Journal of Food Properties, 22(1), 2019, p. 1511-1526. doi: 10.1080/10942912.2019.1656232.

[20] SHAWON, R. et al. Influence of drought stress on bioactive compounds, antioxidant enzymes and glucosinolate contents of Chinese cabbage (Brassica rapa). Food Chemistry, 308, 125657, 2020. doi: 10.1016/j.foodchem.2019.125657. 
[21] ZHANG, C., SUEN, C., YANG, C. and QUEK, S. Antioxidant capacity and major polyphenol composition of teas as affected by geographical location, plantation elevation and leaf grade. Food Chemistry, 244, 2018, p. 109-119. doi: 10.1016/j.foodchem.2017.09.126.

[22] HOSSAIN, M.A. and SHAH, M.D. A study on the total phenols content and antioxidant activity of essential oil and different solvent extracts of endemic plant Merremia borneensis. Arabian Journal of Chemistry, 8, 2015, p. 66-71. doi: https://doi. org/10.1016/j.arabjc.2011.01.007.

[23] KARNAN, R. and SUBRAMANI, K. Antioxidant properties of certain South Indian medicinal plants. Journal of Medicinal Plants Studies, 3(2), 2015, p. 58-60. doi: https://doi.org/10.22271/plants.

[24] KUMAR, P.R. Application of HPLC and ESI-MS techniques in the analysis of phenolic acids and flavonoids from green leafy vegetables (GLVs). Journal of Pharmaceutical Analysis, 7, 2017, p. 349-364. doi: 10.1016/j.jpha.2017.06.005.

[25] RATNADEWI, A. et al. Revealing anti-diabetic potency of medicinal plants of Meru Betiri National Park, Jember - Indonesia. Arabian Journal of Chemistry, 13(1), 2018, p. 1831-1836. doi:10.1016/j. arabjc.2018.01.017.

[26] KAVERY, K. and JEYABALAN, G. Optimization of extraction method and profiling of plant phenolic compounds of Merremia umbellata (hallier. f.) through RP-HPLC. Global Journal for Research Analysis, 8(7), 2019, p. 146-147. doi: https://www. doi.org/10.36106/GJRA.

[27] SHARMA, A. et al. Response of phenylpropanoid pathway and the role of polyphenols in plants under abiotic stress. Molecules, 24(13), 2452, 2019. doi: https://doi.org/10.3390/molecules24132452.

[28] KHAN, R., KHAN, M., SAHREEN, S. and AHMED, M. Evaluation of phenolic contents and antioxidant activity of various solvent extracts of Sonchus asper (L.) Hill. Chemistry Central Journal, 6, 2012, p. 12. doi: 10.1186/1752-153X-6-12.

[29] AJIBOYE, B. et al. Anti-Hyperglycemic and anti-Inflammatory activities of polyphenolic-Rich extract of Syzygium cumini Linn leaves in alloxan-induced diabetic rats. Journal of Evidence-Based Integrative Medicine, 23, 2018, p. 1-8. doi: $10.1177 / 2515690 \times 18770630$.

[30] TAY, Y. et al. Inhibition of carbohydrate hydrolysing enzymes, antioxidant activity and polyphenolic content of Beilschmiedia species extracts. IOP Conference Series: Materials Science and Engineering [online]. 2020. Disponible: https://iopscience.iop.
org/article/10.1088/1757-899X/716/1/012007 [citado 18 de febrero de 2020].

[31] NATUNGNUY, K., CHAREONSAP, P. and POEAIM, S. Biological activities of the methanolic extracts from two varieties of Dimocarpus longan seeds. International Journal of Agricultural Technology, 14(7), 2018, p. 1505-1514.

[32] AL-DABBAGH, B. et al. Antioxidant and anticancer activities of Trigonella foenum-graecum, Cassia acutifolia and Rhazya stricta. BMC Complementary and Alternative Medicine, 18(1), 2018, p. 240. doi: https://doi.org/10.1186/s12906-018-2285-7.

[33] NETO, F. et al. Otimização agroeconômica da cenoura fertilizada com diferentes doses de jitirana. Revista Ciência Agronômica, 45(2), 2014, p. 305311. doi: 10.1590/S1806-66902014000200011.

[34] CABI, current year. Merremia aegyptia. In: Invasive Species Compendium. Wallingford, UK: CAB International. [online]. 2019. Disponible: https://www.cabi.org/isc/datasheet/119828 [citado 11 de abril de 2020].

[35] COLOMBIA. INSTITUTO DE HIDROLOGÍA, METEOROLOGÍA Y ESTUDIOS AMBIENTALES (IDEAM). Cartas climatológicas mensuales, Aeropuerto Almirante Padilla (Riohacha). Subdirección de meteorología [online]. 2018. Disponible: http://bart.ideam.gov.co/cliciu/rioha/ tabla.htm. [citado 28 de enero de 2018].

[36] NAGAI, M. et al. Sweet potato (Ipomoea batatas L.) leaves suppressed oxidation of low density lipoprotein (LDL) in vitro and in human subjects. Journal of Clinical Biochemistry and Nutrition, 48(3), 2011, p. 203-208. doi: 10.3164/jcbn.10-84.

[37] AUSTRIA. INTERNATIONAL ATOMIC ENERGY AGENCY (IAEA). Quantification of tannins in tree foliage. FAO/IAEA working document. Viena (Austria): 2000, 26 p.

[38] INTERNATIONAL CONFERENCE ON HARMONISATION OF TECHNICAL REQUIREMENTS FOR REGISTRATION OF PHARMACEUTICALS FOR HUMAN USE. ICH Harmonised Tripartite Guideline, validation of analytical procedures: text and methodology Q2(R1). Current Step 4 version, Parent Guideline dated 27 October 1994 (Complementary Guideline on Methodology dated 6 November 1996 incorporated in November 2005). Unión Europea: 2005, 17 p.

[39] FARRUKH, A., IQBAL, A. and ZAFAR, M. Antioxidant and free radical scavenging properties of twelve traditionally used indian medicinal plants. Turkish Journal of Biology, 30, 2006, p. 177-183. 
[40] COLOMBIA. INSTITUTO GEOGRÁFICO AGUSTÍN CODAZI (IGAC). Estudio semidetallado de suelos y zonificación de tierras en la media y baja Guajira: escala 1:25.000 (No. AUD 708). Bogotá (Colombia): IGAC, 2012, $810 \mathrm{p}$.

[41] CHERUIYOT, E. et al. Polyphenols as potential indicators for drought tolerance in tea (Camellia sinensis L.). Bioscience, Biotechnology and Biochemistry, 71(9), 2007, p. 2190-2197. doi: 10.1271/ bbb.70156.

[42] MACHADO, M. et al. Polyphenolic compounds, antioxidant activity and L-phenylalanine ammonia-lyase activity during ripening of olive cv. "Cobrançosa" under different irrigation regimes. Food Research International, 51, 2013, p. 412-421. doi: https://doi.org/10.1016/j.foodres.2012.12.056.

[43] KOBAYASHI, T., KURATA, R. and KAI, Y. Seasonal variation in the yield and polyphenol content of sweet potato (Ipomoea batatas L.) Foliage. The Horticulture Journal, 88(2), 2019, p. 270-275. doi: 10.2503/hortj.UTD-025.

[44] GHARIBI, S. et al. The effect of drought stress on polyphenolic compounds and expression of flavonoid biosynthesis related genes in Achillea pachycephala. Rech.f. Phytochemistry, 162, 2019, p. 90-98. doi: 10.1016/j.phytochem.2019.03.004.

[45] KERMANI, S. et al. Drought stress influenced sesamin and sesamolin content and polyphenolic components in sesame (Sesamum indicum L.) populations with contrasting seed coat colors. Food Chemistry, 289, 2019, p. 360-368. doi: 10.1016/j. foodchem.2019.03.004.

[46] KU, Y. et al. Antioxidant capacities and polyphenols in autumn-growing cultivar of Chinese cabbage (Brassica rapa L. ssp. pekinensis cv. Bulam Plus). European Food Research and Technology, 2019. doi: 10.1007/s00217-019-03294-0.

[47] NOURAEI, S., RAHIMMALEK, M. and SAEIDI, G. Variation in polyphenolic composition, antioxidants and physiological characteristics of globe artichoke (Cynara cardunculus var. scolymus Hayek L.) as affected by drought stress. Scientia Horticulturae, 233, 2018, p. 378385. doi: 10.1016/j.scienta.2017.12.060.

[48] SARKER, U. and OBA, S. Response of nutrients, minerals, antioxidant leaf pigments, vitamins, polyphenol, flavonoid and antioxidant activity in selected vegetable amaranth under four soil water content. Food Chemistry, 252, 2018, p. 72-83. doi: 10.1016/j.foodchem.2018.01.097.
[49] SILVA, D. et al. Chemical composition of Caatinga potential forages species. Tropical and Subtropical Agroecosystems, 18, 2015, p. 267 - 272.

[50] ANGAPPAN, R., ANANTH, D. and THILAGAR, S. Diuretic effect of chlorogenic acid from traditional medicinal plant Merremia emarginata (Burm. F.) and its by product hippuric acid. Clinical Phytoscience, 4, 2018, p. 29. doi: https://doi.org/10.1186/ s40816-018-0088-5.

[51] JEYADEVI, R., ANANTH, D. and SIVASUDHA, T. Hepatoprotective and antioxidant activity of Ipomoea staphylina Linn. Clinical Phytoscience, 5(18), 2019. doi: https://doi.org/10.1186/s40816 $-019-0112-4$

[52] MITHÖFER, A., BOLAND, W. and MAFFEI, M. Chemical ecology of plant-insect interactions. Annual Plant Reviews Online, 34, 2018, p. 261291. doi:10.1002/9781119312994.

[53] WAKABAYASHI, K., OGURO, M., ITAGAKI, T. and SAKAI, S. Floral-induced and constitutive defense against florivory: a comparison of chemical traits in 12 herb species. Plant Ecology, 219(8), 2018, p. 985-997. doi: https://doi.org/10.1007/ s11258-018-0851-1.

[54] MURUKAN, G. and MURUGAN, K. Comparison of phenolic acids and antioxidant activities of young and mature leaves of Tectona grandis $\mathrm{L}$ F. Asian Journal of Pharmaceutical and Clinical Research, 11(1), 2018, p. 60-66. doi: 10.22159/ ajpcr.2018.v11i1.19430.

[55] ABDELKEBIR, R., NAJJAA, H., BEN ARFA, A. and MOHAMED, N. Phytochemical screening, antioxidant and antimicrobial activities of Erodium glaucophyllum (L.) L'Hérit. Journal of Biomedical Sciencies, 7(4) 2018. doi: 10.4172/2254-609X.100092.

[56] SURYANTI, V. et al. Identification of active compounds and antioxidant activity of teak (Tectona grandis) leaves. Biodiversitas, 21(3), 2020, p. 946952. doi: 10.13057/biodiv/d210313.

[57] SMERIGLIO, A. et al. Antioxidant and cytoprotective activities of an ancient Mediterranean citrus (Citrus lumia Risso) albedo extract: microscopic observations and polyphenol characterization. Food Chemistry, 279(1), 2019, p. 347-355. doi:10.1016/j. foodchem.2018.11.138.

[58] GÜLÇIN, I. et al. Sage (Salvia pilifera): determination of its polyphenol contents, anticholinergic, antidiabetic and antioxidant activities. Journal of Food Measurement and Characterization, 2019. doi:10.1007/s11694-019-00127-2. 